\title{
HER2 testing on core needle biopsy specimens from primary breast cancers: interobserver reproducibility and concordance with surgically resected specimens
}

\author{
Hitoshi Tsuda ${ }^{1,4^{*}}$, Masafumi Kurosumi ${ }^{2}$, Shinobu Umemura ${ }^{3}$, Sohei Yamamoto ${ }^{4}$, Takayuki Kobayashi ${ }^{4}$,
} Robert Yoshiyuki Osamura ${ }^{3}$

\begin{abstract}
Background: Accurate evaluation of human epidermal growth factor receptor type-2 (HER2) status based on core needle biopsy (CNB) specimens is mandatory for identification of patients with primary breast cancer who will benefit from primary systemic therapy with trastuzumab. The aim of the present study was to validate the application of HER2 testing with CNB specimens from primary breast cancers in terms of interobserver reproducibility and comparison with surgically resected specimens.
\end{abstract}

Methods: A total of 100 pairs of archival formalin-fixed paraffin-embedded CNB and surgically resected specimens of invasive breast carcinomas were cut into sections. All 100 paired sections were subjected to HER2 testing by immunohistochemistry $(\mathrm{IHC})$ and 27 paired sections were subjected to that by fluorescence in situ hybridization (FISH), the results being evaluated by three and two observers, respectively. Interobserver agreement levels in terms of judgment and the concordance of consensus scores between CNB samples and the corresponding surgically resected specimens were estimated as the percentage agreement and $\kappa$ statistic.

Results: In CNB specimens, the percentage interobserver agreement of HER2 scoring by IHC was 76\% ( $\kappa=0.71)$ for $3 \times 3$ categories $(0-1+$ versus $2+$ versus $3+)$ and $90 \%(\kappa=0.80)$ for $2 \times 2$ categories $(0-2+$ versus $3+)$. These levels were close to the corresponding ones for the surgically resected specimens: $80 \%(\kappa=0.77)$ for $3 \times 3$ categories and $92 \%(\kappa=0.88)$ for $2 \times 2$ categories. Concordance of consensus for HER2 scores determined by IHC between CNB and the corresponding surgical specimens was $87 \%(\kappa=0.77)$ for $3 \times 3$ categories, and $94 \%$ $(\kappa=0.83)$ for $2 \times 2$ categories. Among the 13 tumors showing discordance in the mean $\mathrm{IHC}$ scores between the CNB and surgical specimens, the results of consensus for FISH results were concordant in 11. The rate of successful FISH analysis and the FISH positivity rate in cases with a HER2 IHC score of 2+ differed among specimens processed at different institutions.

Conclusion: It is mandatory to study HER2 on breast cancers, and either CNB or surgical specimen can be used.

\section{Background}

The human epidermal growth factor receptor type-2 (HER2) proto-oncogene (c-erbB-2) is amplified in 15-30\% of human breast cancers, causing overexpression of its protein. HER2 gene amplification and/or protein

\footnotetext{
* Correspondence: hstsuda@ncc.go.jp

'Diagnostic Pathology Section, Clinical Laboratory Division, National Cancer

Center Hospital, 5-1-1 Tsukiji, Chuo-ku, Tokyo 104-0045, Japan

Full list of author information is available at the end of the article
}

overexpression is an indicator of clinical tumor aggressiveness [1-3]. The efficacy of trastuzumab, a humanized anti-HER2 monoclonal antibody, against breast cancers with $H E R 2$ gene amplification and/or protein overexpression has been established in clinical trials for patients with metastatic breast cancer or those with operable primary breast cancer as adjuvant systemic therapies [4-7]. Furthermore, as neoadjuvant therapy for patients with breast cancers showing HER2 amplification and/or 
overexpression, therapies involving a combination of trastuzumab and chemotherapy have been shown to be effective in achieving a complete pathological tumor response $[8,9]$.

HER2 testing comprises immunohistochemistry (IHC) to examine protein overexpression and fluorescence in situ hybridization (FISH) to examine gene amplification. These tests are performed on tissue sections of routinely-processed formalin-fixed, paraffin-embedded tumors. High accuracy is required for these HER2 tests in order to identify patients who would benefit from trastuzumab therapy. For the test algorithm, it is generally recommended that IHC is performed first, and that FISH is added if the result of IHC is equivocal [10-12]. Studies of quality assessment have demonstrated that interobserver agreement levels are high for tumors with an IHC score of 0 or $1+$, or those with a score of $3+$, but that the level is generally low for those with a score of 2+ [13-16]. A higher interobserver agreement level can be achieved with FISH than with IHC, especially for tumors with an IHC score of $2+[13,14,16]$. It has also been shown that the quality of both tests is higher at institutions that perform a larger number of HER2 tests than at those where a smaller number of such tests are performed [17-19].

In recent years, core needle biopsies (CNBs) have been used for the qualitative diagnosis of breast tumors. Because of the prevalence of primary systemic therapies as a standard treatment for primary breast cancers, not only hormone receptor status but also HER2 status are generally assayed from $\mathrm{CNB}$ specimens to test the eligibility of patients for primary systemic therapy with trastuzumab [20]. However, it would be expected that examination of CNB specimens alone might result in a proportion of false-positive and/or false-negative results, because CNB samples represent only part of the tumor, notwithstanding the possible presence of intratumor heterogeneity [21-25]. Furthermore, because of the small volume of $\mathrm{CNB}$ specimens, the interobserver agreement rate of HER2 tests for CNB specimens might be lower than for surgically resected specimens.

Therefore, we examined the levels of interobserver agreement for HER2 status determination in both CNB specimens and corresponding surgically resected specimens from 100 patients with primary breast cancer who had not received primary systemic therapy. We compared the HER2 protein status determined by IHC between the CNB specimens and surgically resected specimens of the same tumor. We also compared the HER2 protein status determined by IHC, with the HER2 gene status determined by FISH, in CNB specimens and in the corresponding surgically resected specimens. On the basis of these results, we evaluated the utility and challenges of HER2 testing within CNB specimens.

\section{Methods}

\section{Tissue samples}

We examined 100 paired samples of invasive breast carcinoma obtained by $\mathrm{CNB}$ and surgical resection from patients treated at Saitama Cancer Center, Ina, Saitama (50 cases), Tokai University Hospital, Isehara, Kanagawa (25 cases), and the National Defense Medical College Hospital, Tokorozawa, Saitama (25 cases), Japan. At all three institutions, HER2 testing is performed very frequently for routine diagnostics and/or for studies of quality assessment. Collaborating pathologists in the three institutes were assigned to submit almost equal number of CNB cases of each score (score 0 or $1+, 2+$, and $3+$ ), for the purpose that almost equal number of HER2-negative, equivocal and positive cases were examined in the study. However, these institutional scores were not informed to the pathologists on the central review. Therefore, the cases were not consecutive and there was some selection bias.

None of the patients with these tumors had received neoadjuvant therapy before CNB and surgical resection. At each institution, 4- $\mu \mathrm{m}$-thick sections cut from routinely processed formalin-fixed, paraffin-embedded tissue blocks were subjected to immunohistochemistry and FISH. IHC and FISH assays were performed on parallel slides of the same $\mathrm{CNB}$ /surgically resected specimens. The present study was conducted with approval from the internal review board for ethical issues of the National Defense Medical College. Informed consent had been acquired from each patient for the purpose of general research use of surgically resected tissues, and the requirement for informed consent for the present study was waived by the internal review board according to the guideline of ethical issues for epidemiologic studies by the Ministry of Health, Labor and Welfare and the Ministry of Education, Culture, Sports, Science and Technology, Japan.

\section{Immunohistochemistry}

Expression of HER2 oncoprotein was examined using HercepTest II (Dako, Glostrup, Denmark) in the Dako Japan Central Laboratory, Tokyo, Japan. Deparaffinization, antigen retrieval, and immunohistochemistry were performed to $100 \mathrm{CNB}$ and 100 paired surgically resected tumor sections in accordance with the manufacturer's instructions using an Autostainer Plus (Dako) [16].

Three experienced (for $>25$ years) pathologists (M.K., S.U., H.T.), being blinded from institutional IHC results or the present FISH results, independently evaluated the results of IHC, and assigned a score of 0 (no staining), 1 + (weak, incomplete membrane staining in any proportion of tumor cells), 2+ (complete membrane staining that is either nonuniform or weak in intensity but with 
obvious circumferential distribution in at least $10 \%$ of tumor cells, or invasive tumors show intense, complete membrane staining of $30 \%$ or fewer tumor cells), or $3+$ (uniform, intense membrane staining of $>30 \%$ of invasive tumor cells) in accordance with the guidelines of the American Society of Clinical Oncology (ASCO)/ College of American Pathologists (CAP) [10]. Scores of 0 and $1+$ were categorized as IHC-negative, a score of $2+$ was categorized as equivocal, and a score of $3+$ was categorized as overexpression (positive). If the score assigned by three observers differed among the three categories, the majority scores were acquired as consensus judgments. When the judgments of the three observers differed from each other, the median value was acquired as the representative score.

\section{FISH}

FISH was performed on all $100 \mathrm{CNB}$ specimens and on 27 surgically resected specimens for which the consensus judgment of the IHC result differed from that of the $\mathrm{CNB}$ result and/or was discordant among the three observers.

FISH was performed manually using a PathVysion HER-2 DNA probe kit (Abbott Molecular, Wiesbaden, Germany) in accordance with the manufacturer's instructions [13] at the Department of Basic Pathology, National Defense Medical College, Tokorozawa, Saitama, Japan. The slides were visualized using a Leica DMR fluorescence microscope (Leica, Cambridge, UK). Two observers (S.Y. or T.K. and H.T.), being blinded from IHC results and FISH results of the corresponding $\mathrm{CNB}$ or surgical resection specimen, counted the signals for HER2 and CEP17 on a total of 40 cancer cell nuclei. The total number of $H E R 2$ signals was divided by the total number of CEP17 signals on 40 nuclei, and the HER2/ CEP17 ratio was calculated. HER2 gene amplification was judged as positive, equivocal, and negative if the HER2/ CEP17 ratio was more than $2.2,1.8$ to 2.2 , and less than 1.8 , respectively [10]. When a tumor was judged as equivocal, 40 additional nuclei in another tumor area on invasion were counted again by two observers. If the HER2/ $C E P 17$ ratio was 2.0 or higher in the re-test, the tumor was finally judged as having HER2 amplification.

\section{Interobserver agreement}

With regard to IHC and FISH, interobserver agreement of judgments and the concordance of consensus scores between the CNB and corresponding surgically resected specimens were estimated in terms of percentage agreement and the $\kappa$ statistic. The percentage of agreement was calculated as follows:

(Number of tumors to which the three observers assigned an identical score/total number of tumors) $x$ 100
The level of agreement was categorized as almost perfect, substantial, moderate, fair, and slight when the $\kappa$ value was $>0.80,>0.60-0.80,>0.40-0.60,>0.20-0.40$, and $0-0.20$, respectively $[26,27]$.

\section{Results}

\section{Interobserver agreement for IHC and FISH}

For CNB specimens, the percentage interobserver agreement for HER 2 scores determined by IHC was $76 \%$ for $3 \times 3$ categories $(0-1+$ vs $2+$ vs $3+)$ and $90 \%$ for $2 \times 2$ categories $(0,1+$ or $2+$ vs $3+)$. In terms of the $\kappa$ statistic, the interobserver agreement levels were substantial for the $3 \times 3$ categories $(\kappa=0.71, \sigma=0.065)$ and the $2 \times 2$ categories $(\kappa=0.80, \sigma=0.075)$. The 24 tumors for which judgment discordance arose for $3 \times 3$ categories are shown in Table 1. All of these tumors showed discordance in one score, and none showed a difference in 2 scores.

Table 1 Core needle biopsy tumor specimens for which interobserver disagreement arose regarding the results of HER2 immunohistochemistry

\begin{tabular}{|c|c|c|c|c|c|}
\hline \multirow[t]{2}{*}{ Code } & \multirow{2}{*}{$\begin{array}{l}\text { Final } \\
\text { score }\end{array}$} & \multicolumn{3}{|c|}{ IHC score } & \multirow{2}{*}{$\begin{array}{l}\text { FISH (HER2/ } \\
\text { CEP17) }\end{array}$} \\
\hline & & $\begin{array}{l}\text { Observer } \\
\text { A }\end{array}$ & $\begin{array}{l}\text { Observer } \\
\text { B }\end{array}$ & $\begin{array}{l}\text { Observer } \\
\mathrm{C}\end{array}$ & \\
\hline B45 & $1+$ & $1+$ & $1+$ & $2+$ & $-(0.96)$ \\
\hline B78 & $1+$ & $1+$ & $1+$ & $2+$ & - (0.99) \\
\hline B24 & $1+$ & $2+$ & $1+$ & $1+$ & $-(1.01)$ \\
\hline B82 & $1+$ & $2+$ & $1+$ & $1+$ & $-(1.02)$ \\
\hline B79 & $1+$ & $2+$ & $1+$ & $1+$ & $-(1.05)$ \\
\hline B99 & $1+$ & $1+$ & $1+$ & $2+$ & - (1.07) \\
\hline B36 & $1+$ & $1+$ & $1+$ & $2+$ & $-(1.11)$ \\
\hline B22 & $2+$ & $2+$ & $1+$ & $2+$ & $-(0.97)$ \\
\hline B69 & $2+$ & $2+$ & $1+$ & $2+$ & $-(1.04)$ \\
\hline B43 & $2+$ & $2+$ & $1+$ & $2+$ & - (1.09) \\
\hline B86 & $2+$ & $2+$ & $1+$ & $2+$ & - (1.39) \\
\hline B29 & $2+$ & $2+$ & $2+$ & $1+$ & $-(1.42)$ \\
\hline B100 & $2+$ & $2+$ & $1+$ & $2+$ & $+(2.38)$ \\
\hline B16 & $2+$ & $3+$ & $2+$ & $2+$ & $+(2.56)$ \\
\hline B97 & $2+$ & $2+$ & $1+$ & $2+$ & $+(4.56)$ \\
\hline B52 & $2+$ & $3+$ & $2+$ & $2+$ & $+(5.44)$ \\
\hline B91 & $2+$ & $2+$ & $2+$ & $3+$ & $+(6.80)$ \\
\hline B90 & $2+$ & $3+$ & $2+$ & $2+$ & $+(10.38)$ \\
\hline B102 & $2+$ & $3+$ & $2+$ & $2+$ & $+(12.62)$ \\
\hline B50 & $3+$ & $3+$ & $3+$ & $2+$ & $+(5.11)$ \\
\hline B7 & $3+$ & $3+$ & $2+$ & $3+$ & $+(8.83)$ \\
\hline B96 & $3+$ & $3+$ & $2+$ & $3+$ & $+(9.02)$ \\
\hline B95 & $3+$ & $3+$ & $2+$ & $3+$ & $+(10.92)$ \\
\hline B27 & $3+$ & $3+$ & $3+$ & $2+$ & $+(12.50)$ \\
\hline
\end{tabular}

The HER2 score was assigned according to the 2007 ASCO/CAP guideline [10]. The majority score was assigned as the final score for the tumor. $\mathrm{FISH}$, fluorescence in situ hybridization; IHC, immunohistochemistry 
For surgically resected specimens, the percentage interobserver agreement for HER2 scores determined by IHC was $80 \%$ for the $3 \times 3$ categories and $92 \%$ for the $2 \times 2$ categories. In terms of the $\kappa$ statistic, the interobserver agreement level was substantial for the $3 \times 3$ categories $(\kappa=0.77, \sigma=0.060)$ and almost perfect for the $2 \times 2$ categories $(\kappa=0.88, \sigma=0.051)$. The 20 tumors for which judgment discordance arose for $3 \times 3$ categories are shown in Table 2. All of these tumors showed discordance in one score, and none showed a difference in 2 scores.

The consensus score for HER2 expression determined by IHC was 0 or $1+, 2+$, and $3+$ in 60,19 , and 21 tumors, respectively, in CNB specimens, and in 60, 15, and 25 tumors, respectively, in surgically resected specimens.

FISH was successful in 99 of the 100 CNB specimens. By the first examinations, 96 tumors (97\%) were judged to have the same score by two observers. Because three other tumors were judged differently by two observers and the average of the two judgments was within the range of equivocal, they were subjected to a re-count:

Table 2 Surgically resected tumor specimens for which interobserver disagreement arose regarding the results of HER2 immunohistochemistry

\begin{tabular}{|c|c|c|c|c|c|}
\hline \multirow[t]{2}{*}{ Code } & \multirow{2}{*}{$\begin{array}{l}\text { Final } \\
\text { score }\end{array}$} & \multicolumn{3}{|c|}{ IHC score } & \multirow{2}{*}{$\begin{array}{l}\text { FISH (HER2/ } \\
\text { CEP17) }\end{array}$} \\
\hline & & $\begin{array}{l}\text { Observer } \\
\text { A }\end{array}$ & $\begin{array}{l}\text { Observer } \\
\text { B }\end{array}$ & $\begin{array}{l}\text { Observer } \\
\text { C }\end{array}$ & \\
\hline S54 & $0+$ & 0 & $2+$ & 0 & $-(0.92)$ \\
\hline S17 & $1+$ & $1+$ & $1+$ & $2+$ & NA \\
\hline S26 & $1+$ & $1+$ & $1+$ & $2+$ & $-(0.68)$ \\
\hline S78 & $1+$ & $1+$ & $1+$ & $2+$ & $-(0.82)$ \\
\hline S79 & $1+$ & $2+$ & $1+$ & $1+$ & - $(0.89)$ \\
\hline S76 & $1+$ & $2+$ & $1+$ & $1+$ & - (0.95) \\
\hline S82 & $1+$ & $2+$ & $1+$ & $1+$ & $-(0.97)$ \\
\hline S77 & $1+$ & $2+$ & $1+$ & $1+$ & - (1.47) \\
\hline S23 & $2+$ & $2+$ & $1+$ & $2+$ & $-(0.47)$ \\
\hline S22 & $2+$ & $2+$ & $1+$ & $2+$ & - $(0.92)$ \\
\hline S16 & $2+$ & $3+$ & $2+$ & $2+$ & - (1.03) \\
\hline S84 & $2+$ & $2+$ & $1+$ & $2+$ & $-(1.13)$ \\
\hline S67 & $2+$ & $2+$ & $1+$ & $2+$ & $+(2.24)$ \\
\hline S97 & $2+$ & $3+$ & $2+$ & $2+$ & $+(3.89)$ \\
\hline S103 & $2+$ & $3+$ & $2+$ & $2+$ & $+(7.75)$ \\
\hline S91 & $2+$ & $3+$ & $2+$ & $2+$ & $+(7.77)$ \\
\hline S94 & $3+$ & $3+$ & $3+$ & $2+$ & $+(3.61)$ \\
\hline S52 & $3+$ & $3+$ & $3+$ & $2+$ & $+(5.09)$ \\
\hline S42 & $3+$ & $3+$ & $3+$ & $2+$ & $+(9.24)$ \\
\hline S18 & $3+$ & $3+$ & $3+$ & $2+$ & $+(12.40)$ \\
\hline
\end{tabular}

The HER2 score was assigned according to the 2007 ASCO/CAP guideline [10]. The majority score was assigned as the final score for the tumor. NA, not available because FISH was not successful.

$\mathrm{FISH}$, fluorescence in situ hybridization; IHC, immunohistochemistry for two tumors, the second judgments also differed between two observers, being positive and equivocal respectively, but the average of the judgments of HER2/ CEP17 ratio exceeded 2.20, so they were finally judged positive. For the other, the second judgments were commonly positive (Table 3 ). By FISH, HER2 gene amplification was finally judged positive in $33 \mathrm{CNB}$ specimens (33\%) but negative in 66 (67\%).

A total of 27 surgically resected tumors were subjected to FISH. Among the 25 surgical specimens for which FISH was successful, only one (no. 67) was judged differently by two observers, being positive and equivocal respectively. However, the average of the two judgments was within the range of positive $(H E R 2 / C E P 17$ ratio = 2.236 ), the case was judged as positive without re-evaluation (Table 3). In total, HER2 gene amplification in surgically resected specimens was positive in $12(48 \%)$ and negative in 13 (52\%).

FISH was not successful in three specimens: one CNB and two surgically resected specimens. All three of these specimens were processed in institution A.

\section{Comparison of IHC and FISH test results between CNB and surgically resected specimens}

Concordance in consensus HER2 IHC scores between $\mathrm{CNB}$ and the corresponding surgically resected specimens was $87 \%$ for $3 \times 3$ categories, and $94 \%$ for $2 \times 2$ categories (Table 4). The $\kappa$ statistic indicated that their concordance was substantial for the $3 \times 3$ categories $(\kappa=0.77, \sigma=0.045)$ and almost perfect for the $2 \times 2$ categories $(\kappa=0.83, \sigma=0.038)$. Representative concordant cases are presented in Figure 1.

Table 3 Tumor specimens for which interobserver disagreement arose regarding the results of fluorescence in situ hybridization

\begin{tabular}{|c|c|c|c|c|c|c|c|}
\hline \multicolumn{8}{|c|}{ CNB specimens } \\
\hline \multirow[t]{2}{*}{ Code } & \multirow[t]{2}{*}{$\begin{array}{l}\text { Final } \\
\text { judgment }\end{array}$} & \multicolumn{3}{|c|}{$\begin{array}{l}\text { HER2/CEP17 by } 1 \mathrm{st} \\
\text { counts }\end{array}$} & \multicolumn{3}{|c|}{$\begin{array}{l}\text { HER2/CEP17 by } 2 \text { nd } \\
\text { counts }\end{array}$} \\
\hline & & $\begin{array}{r}\text { Obs. } \\
\text { A }\end{array}$ & $\begin{array}{r}\text { Obs. } \\
\text { B }\end{array}$ & Average & $\begin{array}{r}\text { Obs. } \\
A\end{array}$ & $\begin{array}{r}\text { Obs. } \\
\text { B }\end{array}$ & Average \\
\hline B61 & Amplification & 2.71 & 1.55 & 2.13 & 2.55 & 1.88 & 2.22 \\
\hline B62 & Amplification & 2.35 & 1.88 & 2.12 & 2.44 & 2.07 & 2.26 \\
\hline B87 & Amplification & 1.94 & 2.44 & 2.19 & 3.50 & 2.38 & 2.44 \\
\hline \multicolumn{8}{|c|}{ Surgically resected specimens } \\
\hline \multirow[t]{2}{*}{ Code } & $\begin{array}{l}\text { Final } \\
\text { judgment }\end{array}$ & \multicolumn{3}{|c|}{$\begin{array}{l}\text { HER2/CEP17 by } 1 \text { st } \\
\text { counts }\end{array}$} & \multicolumn{3}{|c|}{$\begin{array}{l}\text { HER2/CEP17 by } 2 \mathrm{nd} \\
\text { counts }\end{array}$} \\
\hline & & $\begin{array}{l}\text { Obs. } \\
\text { A }\end{array}$ & $\begin{array}{l}\text { Obs. } \\
\text { B }\end{array}$ & Average & $\begin{array}{l}\text { Obs. } \\
\text { A }\end{array}$ & $\begin{array}{l}\text { Obs. } \\
\text { B }\end{array}$ & Average \\
\hline$S 67$ & Amplification & 2.67 & 1.81 & 2.24 & ND & ND & 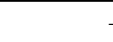 \\
\hline
\end{tabular}

HER2 amplification was defined as positive, equivocal, and negative when the HER2/CEP17 ratio was more than 2.2 , between 1.8 and 2.2, and less than 1.8 , respectively. For the surgically resected specimen, the average of the HER2/ CEP17 ratio (2.24) between observers (2.67 and 1.82) was adopted, because the second count was not done (ND). Obs., observer 


\begin{tabular}{|c|c|c|}
\hline \multirow{4}{*}{$\begin{array}{l}3 \text { categories ( } 0 \text { or } 1+\text { vs. } 2+\text { vs. } 3+\text { ) } \\
\text { HER2 score for surgically resected specimens }\end{array}$} & & \\
\hline & \multicolumn{2}{|c|}{ Number of tumors } \\
\hline & \multicolumn{2}{|c|}{$\begin{array}{c}\text { HER2 score for CNB } \\
\text { specimens } \\
\end{array}$} \\
\hline & 0 or $1+$ & $2+3+$ \\
\hline 0 or $1+$ & 56 & 3 \\
\hline $2+$ & 4 & 11 \\
\hline $3+$ & 0 & $5 \quad 20$ \\
\hline \multicolumn{3}{|c|}{$\%$ agreement $=87 \%, \kappa=0.77$, standard deviation $(\sigma)=0.045$} \\
\hline \multicolumn{3}{|c|}{2 categories $(0,1+$. or $2+$ vs. $3+)$} \\
\hline \multirow[t]{3}{*}{ HER2 score for surgically resected specimens } & \multicolumn{2}{|c|}{ Number of tumors } \\
\hline & \multicolumn{2}{|c|}{$\begin{array}{l}\text { HER2 score for CNB } \\
\text { specimens }\end{array}$} \\
\hline & $0,1+$ or $2+$ & $3+$ \\
\hline $0,1+$, or $2+$ & 74 & 1 \\
\hline $3+$ & 5 & 20 \\
\hline$\%$ agreement $=94 \%, \kappa=0.83, \sigma=0.038$ & & \\
\hline
\end{tabular}

The judgments were performed according to the 2007 ASCO/CAP guideline.

Among the 60 tumors with a score of 0 or $1+$ for CNB specimens, 56 (93\%) were also judged to have a score of 0 or $1+$ but the other four were scored $2+$ for the surgically resected specimens. Among the 21 tumors with a score of $3+$ for CNB specimens, 20 (95\%) were also judged to have a score of $3+$ and only one (5\%) was scored 0 for the surgically resected specimens. In contrast, among the 19 tumors with a score of $2+$ for CNB specimens, only 11 (58\%) were also scored $2+$ for the surgically resected specimens. In three and five of these 19 cases, scores of 0 or $1+$ and $3+$ were assigned, respectively, for the surgically resected specimens.

Overall, consensus HER2 IHC scores showed discordance between CNB and surgically resected specimens in 13 of 100 cases (Table 5). The discordance may have been attributable to intratumor heterogeneity, suboptimal processing of the specimens, and/or the borderline nature of the tumor. The borderline nature means that the state of HER2 expression was borderline between 1+ and $2+$ or between $2+$ and $3+$, namely, the conditions that it was difficult to judge whether the entirely circumscribing membrane immunoreactivity of the HER2 was moderate $(2+)$ or strong $(3+)$, or whether the weak membrane HER2 immunoreactivity was entirely $(2+)$ or incompletely $(1+)$ circumscribing the membrane.

In at least five tumors (Nos. 23, 24, 54, 84, 92), the discordance appeared to have been due to intratumor heterogeneity. In case 54 , to the surgically resected specimen, all three observers gave a score of $3+$ because $>30 \%$ of cancer cells showed strong membrane staining. In contrast, in the CNB specimen, the percentage of cancer cells showing strong membrane staining was around $10 \%$, and two observers gave a score of 0 , and the other gave one of 2+ (Figure 2A). In cases 23, 24, and 84 , there appeared to be intratumor heterogeneity ranging between areas of moderate, and no or weak, HER2 staining (2+ vs 0 or $1+$ ) (Figure 2B). In case 92, the HER2 score for the predominant intraductal component was uniformly $3+$, but in the focus of invasive carcinoma, the immunoreaction was weaker (Figure 2C). In this case, we performed FISH on the part including both invasive carcinoma and non-invasive components.

In 4 cases (Nos. 52, 90, 94 and 102), the HER2 score was $2+$ for the CNB specimen but $3+$ for the surgical specimen (Figure 2D). In these cases, the membrane HER2 immunoreaction in CNB specimens was weaker and less continuous than that in surgically resected specimens. In 5 other cases (Nos. 10, 43, 52, 67 and 69), the intensity of the HER2 immunoreaction differed between the CNB and surgically resected specimens, but the immunoreaction pattern in the surgically resected specimens was uniform. Therefore, suboptimal processing of CNB specimens or long-term fixation of surgically resected specimens might have been partly responsible for the difference.

Interobserver disagreement regarding the HER2 score arose for either CNB specimens or surgically resected specimens in 11 of these 13 cases. Possible reasons for this may have been the borderline nature of the tumors, preanalytical factors, and/or inratumor heterogeneity (e.g., No. 54) (Table 5).

In $23(92 \%)$ of the 25 tumors for which HER2 FISH was performed, consensus judgments regarding HER2 gene status were concordant between the CNB and the surgically resected specimens, being amplified in 11 and not amplified in 12 (Table 6). In the other two cases, the consensus judgments were discordant between the $\mathrm{CNB}$ and the surgically resected specimens: In one (no. 67), the CNB specimen showed an IHC score of $1+$ with a HER2/CEP17 ratio of 0.84 , whereas the surgically resected specimen showed an IHC score of $2+$ with a HER 2/CEP17 ratio of 2.24. As mentioned above, the case was judged differently by two observers, but the average of the judgments made a score of positive (Table 3). In the other, both the CNB and surgically resected specimens showed an IHC score of $2+$, and the HER 2/CEP17 ratio was 1.03 and 2.56, respectively (No. 16). For that case, there was no disagreement in HER2 score of CNB and surgically resected specimens by IHC.

\section{Correlation between IHC and FISH results}

Among the $100 \mathrm{CNB}$ specimens, the percentage of HER2 gene amplification was $95 \%$ (20 of 21), 58\% (11 of $19)$, and $3 \%$ ( 2 of 60$)$ for HER2 IHC scores of $3+, 2+$, and 0 or $1+$, respectively (Table 7 ). With regard to 

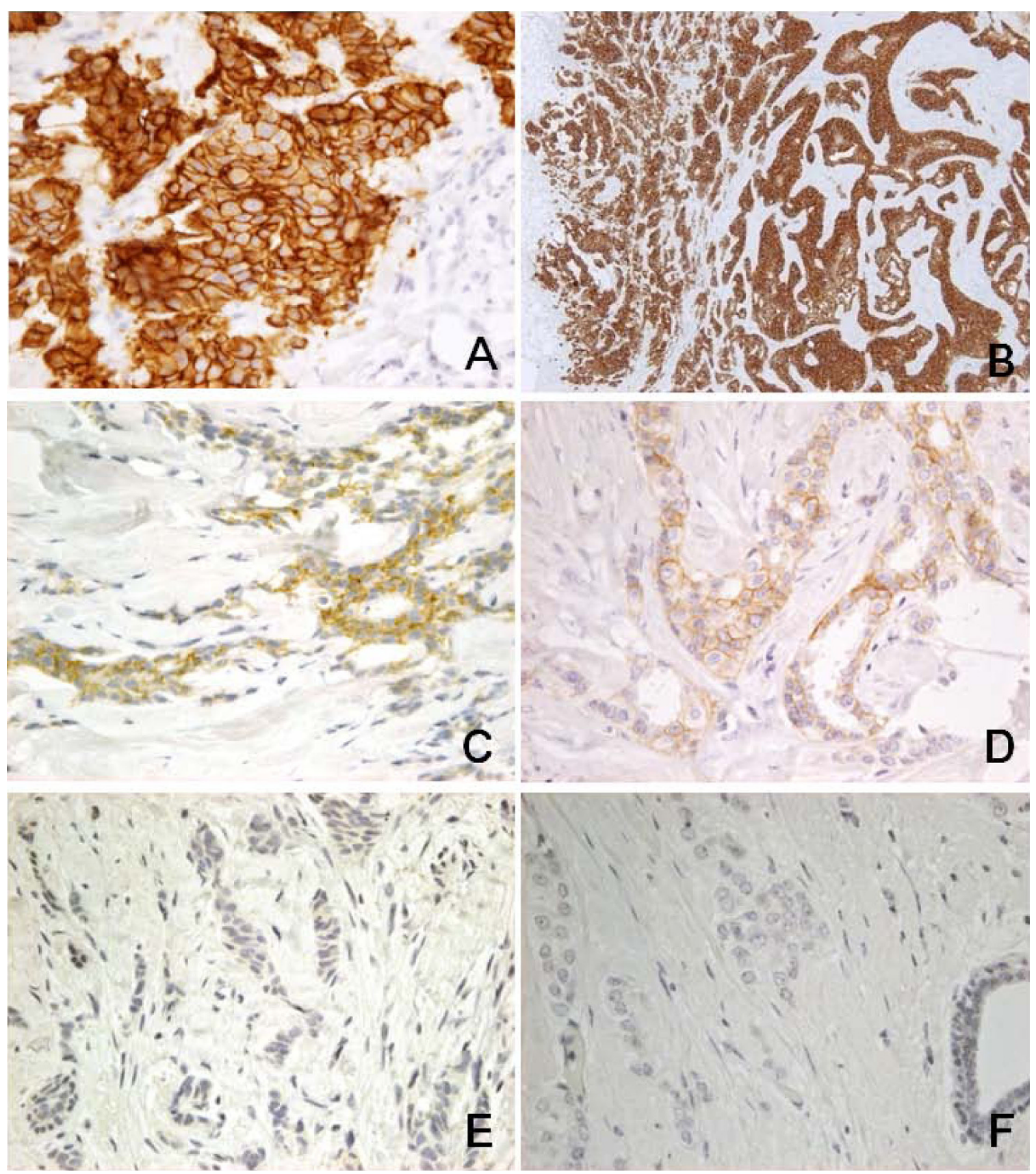

Figure 1 Cases with concordant judgment of HER2 score between CNB and surgically resected specimens. A-B. Case 3: HER2 sCore for both the CNB specimen (A) and the surgically resected specimen (B) was 3+. C-D. Case 22: HER2 score for both the CNB specimen (C) and the surgically resected specimen (D) was 2+. E-F. Case 2: HER2 score for both the CNB specimen (E) and the surgically resected specimen (F) was 0. Immunoperoxidase reaction, original magnification $\times 200$.

tumors with an IHC score of $2+$, the ratio of HER2 gene amplification differed between tumor specimens from different institutions. In tumor specimens from institutions $\mathrm{A}$ and $\mathrm{B}$, the rates of HER2 gene amplification were $20 \%$ ( 1 of 5 ) and 33\% ( 1 of 3 ), respectively, but in specimens from institute $\mathrm{C}$, the rate was as high as $82 \%$ (9 of 11).
For the 25 surgically resected specimens successfully subjected to FISH, the percentage of HER2 gene amplification was $100 \%$ (10 of 10), 43\% (3 of 7), and $0 \%$ (0 of 8) for HER2 IHC scores of $3+, 2+$, and 0 or $1+$, respectively (Table 7). In tumor specimens from institution $\mathrm{A}$, the rate of HER 2 gene amplification in IHC $2+$ cases was $0 \%$ ( 0 of 3 ), whereas in specimens from institution 
Table 513 tumors for which interobserver disagreement arose regarding the results of immunohistochemistry.

\begin{tabular}{llllll}
\hline Code No. & \multicolumn{3}{c}{ Immunohistochemistry } & \multicolumn{2}{c}{ FISH } \\
\cline { 2 - 6 }- & CNB & Surgery & Interpretation & CNB & Surgery \\
\hline 84 & $0(0 / 0 / 0)$ & $2+(\underline{(2 / 1 / 2)})$ & Processing, hetero & Neg & Neg \\
23 & $1+(1 / 1 / 1)$ & $2+(\underline{(2 / 1 / 2)}$ & Heterogeneity & Neg & Neg \\
24 & $1+(\underline{(2 / 1 / 1)}$ & $2+(2 / 2 / 2)$ & Heterogeneity & Neg & NA \\
67 & $1+(\underline{(1 / 0 / 1)})$ & $2+(\underline{(2 / 1 / 2)})$ & Processing, border & Neg & Pos \\
10 & $2+(2 / 2 / 2)$ & $1+(1 / 1 / 1)$ & Processing & Neg & Neg \\
43 & $2+(\underline{(2 / 1 / 2)})$ & $0(0 / 0 / 0)$ & Processing & Neg & Neg \\
69 & $2+(\underline{(2 / 1 / 2)})$ & $1+(1 / 1 / 1)$ & Processing, border & Neg & Neg \\
52 & $2+(\underline{3 / 2 / 2)})$ & $3+(\underline{3 / 3 / 2})$ & Processing, border & Pos & Pos \\
90 & $2+(\underline{3 / 2 / 2)}$ & $3+(3 / 3 / 3)$ & Processing, border & Pos & Pos \\
92 & $2+(2 / 2 / 2)$ & $3+(3 / 3 / 3)$ & Predominantly DCIS & Pos & Pos \\
94 & $2+(2 / 2 / 2)$ & $3+(3 / 3 / 2)$ & Processing, border & Pos & Pos \\
102 & $2+(\underline{3 / 2 / 2)}$ & $3+(3 / 3 / 3)$ & Processing, border & Pos & Pos \\
54 & $3+(3 / 3 / 3)$ & $0(\underline{(0 / 2 / 0)}$ & Heterogeneity & Neg & Neg \\
\hline
\end{tabular}

FISH, fluorescence in situ hybridization; NA, Data were not available; neg, negative; pos, positive; parenthesis, judgments of scores by three observers (Underlines indicate interobserver disagreement.)

$\mathrm{C}$, the rate was $80 \%$ ( 4 of 5 ). No cases with a score of $2+$ were subjected to FISH at institution B.

For CNB specimens, 14 cases showed interobserver disagreement in HER2 IHC scores of between 1+ and 2+, and, of these, seven tumors each were finally scored as 1 + and $2+$. Among these tumors, HER2 gene amplification was detected in $0(0 \%)$ and $2(29 \%)$, respectively. The other 10 cases showed interobserver disagreement in HER2 IHC scores of between $2+$ and $3+$, and, of these, five tumors each were finally scored as $2+$ and $3+$. HER2 gene amplification was detected in all of these tumors (Table 1).

For surgically resected specimens, 12 cases showed interobserver disagreement between IHC scores of $0 / 1+$ and $2+$, and eight and four tumors were finally scored as $0 / 1+$ and $2+$, respectively. Among these tumors, HER2 gene amplification was detected in $0(0 \%)$ and 1 (25\%), respectively. The other eight showed interobserver discordance between IHC scores of $2+$ and $3+$, and four tumors each were finally scored as $2+$ and $3+$. Among these tumors, HER2 gene amplification was detected in $3(75 \%)$ and $4(100 \%)$, respectively (Table 2$)$.

In the 12 tumors showing discordance of the IHC score for HER2 expression between the CNB and surgically resected specimens, and for which the FISH assay was successful, judgments for the FISH results were concordant in 11 (92\%) (Table 5).

\section{Discussion}

From the viewpoint of interobserver agreement level, the percentage agreement levels and $\kappa$ values among the three observers with regard to the IHC test results were similar between the data for CNB specimens and those for surgically resected specimens. Furthermore, the present study also clarified that the results of IHC for HER2 were mostly concordant between the CNB specimen and the surgically resected specimen from an identical invasive breast cancer. These results indicated that the CNB specimens were of adequate quality for the evaluation of HER2 status by IHC, and that the IHC scores obtained for the CNB specimens were mostly representative of the HER2 IHC scores for the entire tumor specimen. Therefore, HER2 testing using IHC for CNB specimens appeared to be valid for a majority of primary breast cancers. The present results were similar to the very first studies done by Chivukula et al. that stated as CNB a better sample [25].

Previous reports have indicated that concordance of HER2 IHC scores between CNB and corresponding excisional biopsy/surgically resected specimens was $87-98.8 \%$ [21-23,28]. The introduction of FISH analysis has improved the concordance rate for HER2 status [22,25]. In the study by Apple et al., the concordance rate of $87 \%$ for IHC was improved to $92 \%$ by FISH [22]. Intratumor heterogeneity for HER2 amplification was reported to be present in $13 \%$ of tumors of an IHC score of $3+$, and was especially higher in those with low-grade amplification (ratio $>2.2$ to $<4.0$ ) [24]. For tumors with an IHC score of $2+$, the incidence of intratumor heterogeneity in HER2 scores was also relatively frequent (14\%), but FISH analysis of CNB specimens almost completely resolved the issue of heterogeneous HER2 expression [25].

Some studies had suggested that the validity of IHC score $3+$ in core biopsies was limited, reporting high rates of false positives (19.3\%). However, Moelans et al, showed that there was only a slightly higher percentage of IHC $3+$ positivity in biopsies compared to resections, and that did not reach statistical difference [29]. Their results are in line with those in the present study (25\% vs $21 \%$ ). In the present study, it was confirmed that concordance of the HER2 test results was higher for FISH (98\%) than for IHC (87\%). Among the 13 tumors that showed discordance of HER2 IHC scores between the CNB and surgically resected specimens, 11 showed concordance of the results obtained by FISH.

The disagreement in the results obtained with IHC between CNB and surgical specimens appeared to be derived from 1 . intratumor heterogeneity, 2. pre-analytical factors including variations in the duration of fixation and suboptimal tissue processing, and/or 3. borderline tumor properties. To overcome the problem of intratumor heterogeneity in HER2 expression, examination of a large volume of tumor tissue appears to be necessary. To solve the problem of borderline tumor properties in terms of HER2 expression, the introduction of judgment by multiple observers and/or DNA copy analyses might be of value. 


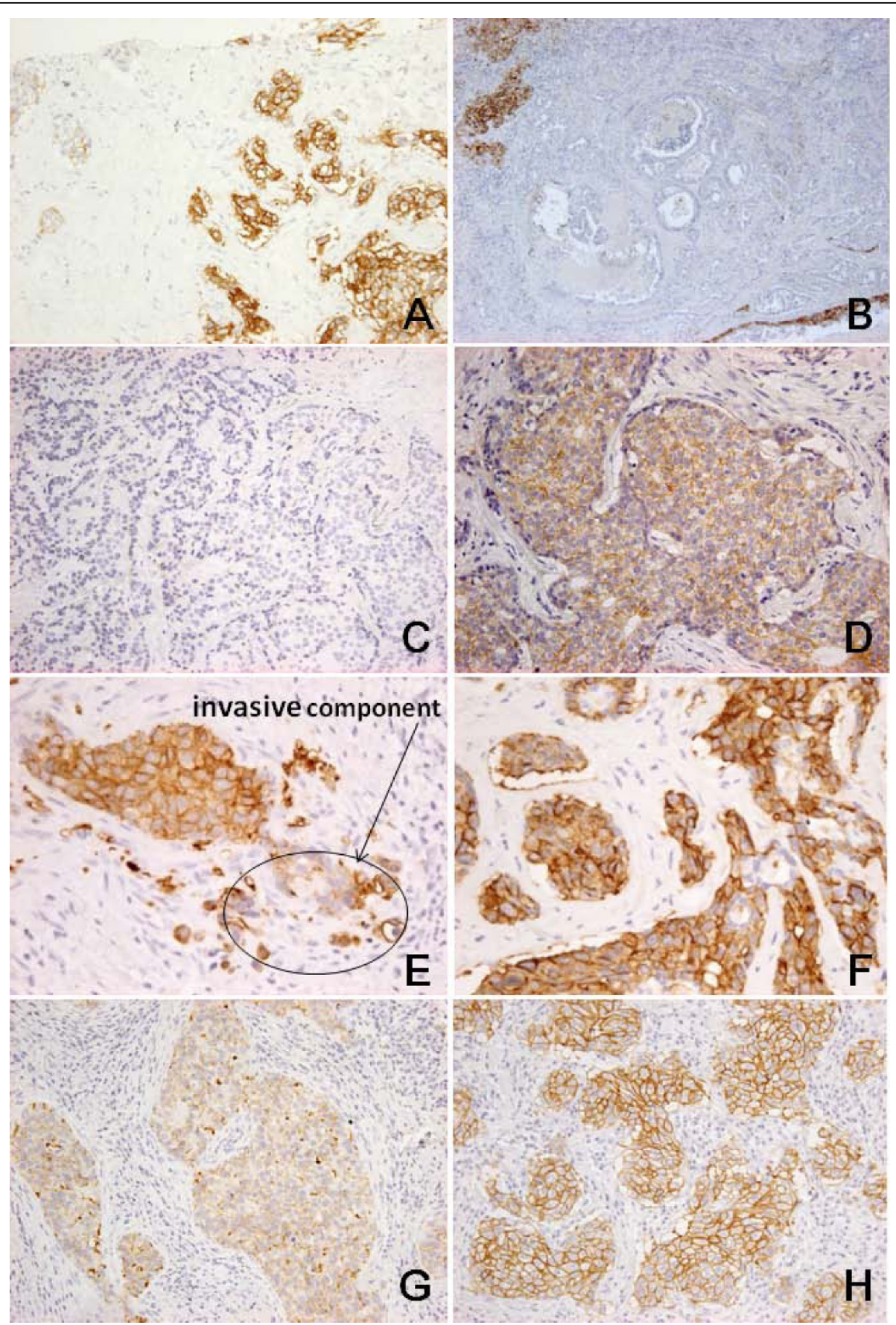

Figure 2 Cases with discordant judgment of HER2 score between CNB and surgically resected specimens. A-B. Case 54: HER2 score was $3+$ for the CNB specimen (A) but 0 for the surgically resected specimen (B). The tumor had heterogeneous HER2 expression, and $>30 \%$ of the area showed a strong membrane immunoreaction in the CNB specimen, whereas most of the area in the surgically resected specimen was HER2-negative. C-D. Case 84: HER2 score was 0 for the CNB specimen (C) but 2+ for the surgically resected specimen (D). This difference might have been due to suboptimal processing of CNB specimens or intratumor heterogeneity. E-F. Case 92: HER2 score was 2+ for the CNB specimen (E) but $3+$ for the surgically resected specimen (F). Because the CNB specimen contained only a small amount of invasive component, the evaluation of HER2 was difficult. G-H. Case 94: HER2 score was 2+ for the CNB specimen (G) but 3+ for the surgically resected specimen (H). Processing of the CNB specimen might have been suboptimal, or the tumor may have been borderline in nature. Immunoperoxidase reaction, original magnification $\times 200$. 
Table 6 Concordance of consensus HER2 FISH results of between core needle biopsy and surgically resected specimens

\begin{tabular}{|c|c|c|c|}
\hline \multirow[t]{3}{*}{ HER2/CEP17 ratio for surgically resected specimens } & \multicolumn{3}{|c|}{ Number of tumors } \\
\hline & \multicolumn{3}{|c|}{ HER2/CEP17 ratio for CNB specimens } \\
\hline & $>2.2$ & 1.8 to 2.2 & $<1.8$ \\
\hline Higher than 2.2 & 11 & 0 & 1 \\
\hline 1.8 to 2.2 & 0 & 0 & 0 \\
\hline$<1.8$ & 1 & 0 & 12 \\
\hline
\end{tabular}

$\%$ agreement $=92 \%$

The judgments were performed according to the 2007 ASCO/CAP guideline. For two tumors, data on CNB and/or surgically resected specimens were not available.

From the present results, the rate of HER2 gene amplification appeared to be low when interobserver discordance was seen between IHC scores of $0 / 1+$ and 2 + , whereas most tumors had gene amplification when there was interobserver discordance between scores of 2 + and 3+. These findings will be helpful for the decision if subsequent FISH should be performed or not. For 2 $+/ 3+$ discrepancies FISH test should always be added because the percentage of HER2 amplification is high. Counting of HER2 gene copies by FISH, chromogenic in situ hybridization (CISH), silver-enhanced in situ hybridization (SISH), or dual-color dual-hapten in situ hybridization (DDISH) would greatly improve the concordance of HER2 status between CNB and surgically resected specimens [30-32].

Table 7 Correlation between consensus immunohistochemistry and FISH results for core needle biopsy and surgically resected specimens

\begin{tabular}{|c|c|c|c|c|}
\hline \multicolumn{5}{|c|}{ Core needle biopsy specimens } \\
\hline & \multicolumn{4}{|c|}{ Number of tumors (\%) } \\
\hline & \multirow[t]{2}{*}{ Total } & \multicolumn{3}{|c|}{ HER2 gene amplification by FISH } \\
\hline & & Positive & Equivocal* & Negative \\
\hline \multicolumn{5}{|c|}{ HER2 IHC score } \\
\hline $3+$ & 21 & $20(95)$ & $0(0)$ & $1(5)$ \\
\hline $2+$ & 19 & $11(58)$ & $0(0)$ & $8(42)$ \\
\hline 0 or $1+$ & 60 & $2(3)$ & $0(0)$ & $58(97)$ \\
\hline Total & 100 & $33(33)$ & $0(0)$ & $67(67)$ \\
\hline \multicolumn{5}{|c|}{ Surgically resected specimens } \\
\hline & \multicolumn{4}{|c|}{ Number of tumors (\%) } \\
\hline & \multirow[t]{2}{*}{ Total } & \multicolumn{3}{|c|}{ HER2 gene amplification by FISH } \\
\hline & & Positive & Equivocal* & Negative \\
\hline \multicolumn{5}{|c|}{ HER2 IHC score } \\
\hline $3+$ & 10 & 10(100) & $0(0)$ & $0(0)$ \\
\hline $2+$ & 7 & $3(43)$ & $0(0)$ & $4(57)$ \\
\hline 0 or $1+$ & 8 & $0(0)$ & $0(0)$ & $8(100)$ \\
\hline Total & 25 & $13(52)$ & $0(0)$ & $12(48)$ \\
\hline
\end{tabular}

*For three tumors, FISH judgment was equivocal by the first counts, but by the second counts, the judgments changed into positive (Table 3). FISH, fluorescence in situ hybridization
Another important factor to be considered is the preanalytical condition of the specimens. In the present study, FISH analysis was sometimes unsuccessful for specimens processed at institution A, whereas FISH was always successful for specimens processed at institutions $\mathrm{B}$ and $\mathrm{C}$. Interview revealed that the suboptimal FISH results obtained at institution $\mathrm{A}$ were attributable to long-term (about 1 week) fixation of the specimens. It was shown that a prolonged formalin fixation could lose FISH amplification and/or yield to unsuccessful test (Hiroi S, Tsuda $\mathrm{H}$ et al, manuscript in preparation) [33].

On the other hand, the localization of the HER2 immunoreaction on the cancer cell membrane was not uniform in CNB specimens from HER2-positive tumors processed at institution $\mathrm{C}$, whereas this was not an evident feature in specimens processed at other institutions or in surgically resected specimens. The reason for this unusual immunoreaction is unclear. It has been shown that quality assessment of HER2 tests is very important for identifying patients who are very likely to benefit from therapy with trastuzumab $[10,34,35]$. For quality assessment, not only improvement of the interobserver agreement level but also standardization of pre-analytical specimen preparation should be taken into consideration.

\section{Conclusion}

We have clarified that CNB specimens showed almost equal reliability to surgically resected specimens for testing of HER2 expression in terms of interobserver agreement levels and concordance with FISH results. In most of specimens with equivocal IHC results, accurate HER2 status was known determined by retesting with FISH. To further improve the reliability of HER2 tests using CNB specimens, it might be useful to sample a larger volume of tumor tissue, to conduct evaluation by multiple observers, and to take measures to improve the preanalytical conditions of the specimens.

List of abbreviations used

CNB: core needle biopsy; FISH: fluorescence in situ hybridization; HER2:

human epidermal growth factor receptor type-2; IHC: immunohistochemistry 


\section{Competing interests}

The authors declare that they have no competing interests.

\section{Authors' contributions}

HT conceived the study, acquired data of IHC and FISH, analyzed data, and prepared manuscript. MK and SU also conceived the study, acquired data of IHC and analyzed data. SY and TK acquired data of FISH and analyzed data. RYO also conceived the study and supervised the analysis. All authors read and approved the final manuscript.

\section{Acknowledgements}

This study was supported in part by a grant-in-aid for Cancer Research from the Ministry of Health, Labor, and Welfare, Japan and a grant-in-aid from the Foundation for Promotion of Defense Medicine, Tokorozawa, Japan. We are grateful to Dr. Yutaka Hatanaka, Department of Medical Science, Dako Japan Inc., Kyoto, for immunohistochemistry and to Ms. Kozue Suzuki, Department of Basic Pathology, National Defense Medical College, Tokorozawa, for FISH. We thank Dr. Futoshi Akiyama, Department of Clinical Pathology, Cancer Institute, Tokyo; Dr. Yutaka Tokuda, Department of Surgery, Tokai University School of Medicine, Isehara; Dr. Masakazu Toi, Department of Breast Surgery, Kyoto University Graduate School of Medicine, Kyoto; Dr. Toru Watanabe, Hamamatsu Oncology Center, Hamamatsu; and Dr. Goi Sakamoto, Sakamoto Memorial Clinic Academy of Breast Pathology, Tokyo, for their critical advice.

\section{Author details}

'Diagnostic Pathology Section, Clinical Laboratory Division, National Cancer Center Hospital, 5-1-1 Tsukiji, Chuo-ku, Tokyo 104-0045, Japan. ${ }^{2}$ Department of Pathology, Saitama Cancer Center Hospital, 818 Komuro, Ina-machi, Kitaadachi-gun, Saitama 362-0806, Japan. ${ }^{3}$ Department of Pathology, Tokai University School of Medicine, Shimokasuya 143, Isehara, Kanagawa 2591193, Japan. ${ }^{4}$ Department of Basic Pathology, National Defense Medical College, 3-2 Namiki, Tokorozawa, Saitama 359-8513, Japan.

Received: 20 February 2010 Accepted: 7 October 2010 Published: 7 October 2010

\section{References}

1. Slamon DJ, Clark GM, Wong SG, Levin WJ, Ullrich A, McGuire WL: Human breast cancer: correlation of relapse and survival with amplification of the HER-2/neu oncogene. Science 1987, 235:177-182.

2. Kallioniemi OP, Kallioniemi A, Kurisu W, Thor A, Chen LC, Smith HS, Waldman FM, Pinkel D, Gray JW: ERBB2 amplification in breast cancer analyzed by fluorescence in situ hybridization. Proc Natl Acad Sci USA 1992, 89:5321-5325.

3. Chia S, Norris B, Speers C, Cheang M, Gilks B, Gown AM, Huntsman D, Olivotto IA, Nielsen TO, Gelmon K: Human epidermal growth factor receptor 2 overexpression as a prognostic factor in a large tissue microarray series of node-negative breast cancers. J Clin Oncol 2008, 26:5697-5704

4. Slamon DJ, Leyland-Jones B, Shak S, Fuchs H, Paton V, Bajamonde A, Fleming T, Eiermann W, Wolter J, Pegram M, Baselga J, Norton L: Use of chemotherapy plus a monoclonal antibody against HER2 for metastatic breast cancer that overexpresses HER2. N Engl J Med 2001, 344:783-792.

5. Romond EH, Perez EA, Bryant J, Suman VJ, Geyer CE Jr, Davidson NE, TanChiu E, Martino S, Paik S, Kaufman PA, Swain SM, Pisansky TM, Fehrenbacher L, Kutteh LA, Vogel VG, Visscher DW, Yothers G, Jenkins RB, Brown AM, Dakhil SR, Mamounas EP, Lingle WL, Klein PM, Ingle JN, Wolmark N: Trastuzumab plus adjuvant chemotherapy for operable HER2-positive breast cancer. N Engl J Med 2005, 353:1673-1684.

6. Piccart-Gebhart MJ, Procter M, Leyland-Jones B, Goldhirsch A, Untch M, Smith I, Gianni L, Baselga J, Bell R, Jackisch C, Cameron D, Dowsett M, Barrios CH, Steger G, Huang CS, Andersson M, Inbar M, Lichinitser M, Láng I, Nitz U, Iwata H, Thomssen C, Lohrisch C, Suter TM, Rüschoff J, Suto T, Greatorex V, Ward C, Straehle C, McFadden E, Dolci MS, Gelber RD: Herceptin Adjuvant (HERA) Trial Study Team. Trastuzumab after adjuvant chemotherapy in HER2-positive breast cancer. N Engl J Med 2005, 353:1659-1672.

7. Joensuu H, Kellokumpu-Lehtinen PL, Bono P, Alanko T, Kataja V, Asola R, Utriainen T, Kokko R, Hemminki A, Tarkkanen M, Turpeenniemi-Hujanen T, Jyrkkiö S, Flander M, Helle L, Ingalsuo S, Johansson $K$, Jääskeläinen $A S$, Pajunen M, Rauhala M, Kaleva-Kerola J, Salminen T, Leinonen M, Elomaa I,
Isola J, FinHer Study Investigators: Adjuvant docetaxel or vinorelbine with or without trastuzumab for breast cancer. N Engl J Med 2006, 354:809-820.

8. Buzdar AU, Valero V, Ibrahim NK, Francis D, Broglio KR, Theriault RL, Pusztai L, Green MC, Singletary SE, Hunt KK, Sahin AA, Esteva F, Symmans WF, Ewer MS, Buchholz TA, Hortobagyi GN: Neoadjuvant therapy with paclitaxel followed by 5 -fluorouracil, epirubicin, and cyclophosphamide chemotherapy and concurrent trastuzumab in human epidermal growth factor receptor 2-positive operable breast cancer: an update of the initial randomized study population and data of additional patients treated with the same regimen. Clin Cancer Res 2007, 13:228-233.

9. Hurley J, Doliny P, Reis I, Silva O, Gomez-Fernandez C, Velez P, Pauletti G, Powell JE, Pegram MD, Slamon DJ: Docetaxel, cisplatin, and trastuzumab as primary systemic therapy for human epidermal growth factor receptor 2-positive locally advanced breast cancer. J Clin Oncol 2006, 24:1831-1838.

10. Wolff AC, Hammond ME, Schwartz JN, Hagerty KL, Allred DC, Cote RJ, Dowsett M, Fitzgibbons PL, Hanna WM, Langer A, McShane LM, Paik S, Pegram MD, Perez EA, Press MF, Rhodes A, Sturgeon C, Taube SE, Tubbs R, Vance GH, van de Vijver M, Wheeler TM, Hayes DF: American Society of Clinical Oncology/College of American Pathologists guideline recommendations for human epidermal growth factor receptor 2 testing in breast cancer. J Clin Oncol 2007, 25:118-145.

11. Carlson RW, Moench SJ, Hammond ME, Perez EA, Burstein HJ, Allred DC, Vogel CL, Goldstein L, Somlo G, Gradishar WJ, Hudis CA, Jahanzeb M, Stark A, Wolff AC, Press MF, Winer EP, Paik S, Ljung BM: NCCN HER2 Testing in Breast Cancer Task Force. HER2 testing in breast cancer: NCCN Task Force report and recommendations. I Natl Compr Canc Netw 2006, 4(Suppl 3):S1-S22.

12. Sauter G, Lee J, Bartlett JM, Slamon DJ, Press MF: Guidelines for human epidermal growth factor receptor 2 testing: biologic and methodologic considerations. J Clin Oncol 2009, 27:1323-1333.

13. Tsuda H, Akiyama F, Terasaki H, Hasegawa T, Kurosumi M, Shimadzu M, Yamamori S, Sakamoto G, Detection of HER-2/neu (c-erbB-2) DNA amplification in primary breast carcinoma: Interobserver reproducibility and correlation with immunohistochemical HER-2 overexpression. Cancer 2001, 92:2965-2974.

14. Press MF, Sauter $G$, Bernstein L, Villalobos IE, Mirlacher M, Zhou JY, Wardeh R, Li YT, Guzman R, Ma Y, Sullivan-Halley J, Santiago A, Park JM, Riva A, Slamon DJ: Diagnostic evaluation of HER-2 as a molecular target: an assessment of accuracy and reproducibility of laboratory testing in large, prospective, randomized clinical trials. Clin Cancer Res 2005, 11:6598-6607.

15. Tsuda H, Sasano H, Akiyama F, Kurosumi M, Hasegawa T, Osamura RY, Sakamoto G: Evaluation of interobserver agreement in scoring immunohistochemical results of HER-2/neu (c-erbB-2) expression detected by HercepTest, Nichirei polyclonal antibody, CB11 and TAB250 in breast carcinoma. Pathol Int 2002, 52:126-134.

16. Umemura S, Osamura RY, Akiyama F, Honma K, Kurosumi M, Sasano H, Toyoshima S, Tsuda H, Rüschoff J, Sakamoto G: What causes discrepancies in HER2 testing for breast cancer? A Japanese ring study in conjunction with the global standard. Am J Clin Pathol 2008, 130:883-891.

17. Roche PC, Suman VJ, Jenkins RB, Davidson NE, Martino S, Kaufman PA Addo FK, Murphy B, Ingle JN, Perez EA: Concordance between local and central laboratory HER2 testing in the breast intergroup trial N9831. J Natl Cancer Inst 2002, 94:855-857.

18. Paik S, Bryant J, Tan-Chiu E, Romond E, Hiller W, Park K, Brown A, Yothers G, Anderson S, Smith R, Wickerham DL, Wolmark N: Real-world performance of HER2 testing-National Surgical Adjuvant Breast and Bowel Project experience. J Natl Cancer Inst 2002, 94:852-854.

19. Reddy JC, Reimann JD, Anderson SM, Klein PM: Concordance between central and local laboratory HER2 testing from a community-based clinical study. Clin Breast Cancer 2006, 7:153-157.

20. Bast RC Jr, Ravdin P, Hayes DF, Bates S, Fritsche H Jr, Jessup JM, Kemeny N, Locker GY, Mennel RG, Somerfield MR: American Society of Clinical Oncology Tumor Markers Expert Panel. 2000 update of recommendations for the use of tumor markers in breast and colorectal cancer: clinical practice guidelines of the American Society of Clinical Oncology. J Clin Oncol 2001, 19:1865-1878. 
21. Usami S, Moriya T, Amari M, Suzuki A, Ishida T, Sasano H, Ohuchi N: Reliability of prognostic factors in breast carcinoma determined by core needle biopsy. Jpn J Clin Oncol 2007, 37:250-255.

22. Apple SK, Lowe AC, Rao PN, Shintaku IP, Moatamed NA: Comparison of fluorescent in situ hybridization HER2/neu results on core needle biopsy and excisional biopsy in primary breast cancer. Mod Pathol 2009, 22:1151-1159.

23. Arnedos M, Nerurkar A, Osin P, A'hern R, Smith IE, Dowsett M: Discordance between core needle biopsy (CNB) and excisional biopsy (EB) for estrogen receptor (ER), progesterone receptor (PgR) and HER2 status in early breast cancer (EBC). Ann Oncol 2009, 20:1948-1952

24. Brunelli M, Manfrin E, Martignoni G, Miller K, Remo A, Reghellin D, Bersani $S$, Gobbo S, Eccher A, Chilosi M, Bonetti F: Genotypic intratumoral heterogeneity in breast carcinoma with HER2/neu amplification: evaluation according to ASCO/CAP criteria. Am J Clin Pathol 2009, 131:678-682.

25. Chivulkula M, Bhargava R, Brufsky A, Surti U, Dabbs DJ: Clinical importance of HER2 immunohistologic heterogeneous expression in core-needle biopsies vs resection specimens for equivocal (immunohistochemical score 2+) cases. Mod Pathol 2008, 21:363-368.

26. Fleiss $\mathrm{JL}$ : Measuring nominal scale agreement among many raters. Psychol Bull 1971, 76:378-382.

27. Landis JR, Koch GG: The measurement of observer agreement for catetgorical data. Biometrics 1977, 33:159-174.

28. D'Alfonso T, Liu YF, Monni S, Rosen PP, Shin SJ: Accurately assessing her$2 /$ neu status in needle core biopsies of breast cancer patients in the era of neoadjuvant therapy: emerging questions and considerations addressed. Am J Surg Pathol 2010, 34:575-581.

29. Moelans $C B$, de Weger RA, van Blokland MT, Ezendam C, Elshof S, Tilanus MG, van Diest PJ: HER-2/neu amplification testing in breast cancer by multiplex ligation-dependent probe amplification in comparison with immunohistochemistry and in situ hybridization. Cell Oncol 2009, 31:1-10.

30. Shousha S, Peston D, Amo-Takyi B, Morgan M, Jasani B: Evaluation of automated silver-enhanced in situ hybridization (SISH) for detection of HER2 gene amplification in breast carcinoma excision and core biopsy specimens. Histopathology 2009, 54:248-253.

31. Wolf V, Große R, Erggelet J, Holzhausen HJ, Hauptmann S, Thomssen C: The reliability of HER2-status determination from core-needle-biopsies and surgical specimens: A comparison of two established test methods (IHC, CISH). J Clin Oncol 2009, 27:15S, (suppl. Abstr. e22141).

32. Gruver AM, Peerwani Z, Tubbs RR: Out of the darkness and into the light: bright field in situ hybridisation for delineation of ERBB2 (HER2) status in breast carcinoma. J Clin Pathol 2010, 63:210-219.

33. Hiroi S, Tsuda H, Oya T, Hayama T, Kawai T, Torikata C: Influence of fixation period on the detection of HER2/neu protein and HER2/neu gene signals. Proc Jpn Soc Pathol 2003, 92:222, (abstract in Japanese).

34. Terry J, Torlakovic EE, Garratt J, Miller D, Köbel M, Cooper J, Bahzad S, Pilavdzic D, O'Malley F, O'Brien AE, SenGupta S, Alport E, Têtu B, Knight B, Pettigrew NM, Berendt R, Wolber R, Trotter MJ, Riddell RH, Gaboury L, Elms F, Magliocco A, Barnes P, Gown AM, Gilks CB: Implementation of a Canadian external quality assurance program for breast cancer biomarkers: an initiative of Canadian Quality Control in immunohistochemistry (clQc) and Canadian Association of Pathologists (CAP) National Standards Committee/Immunohistochemistry. Appl Immunohistochem Mol Morphol 2009, 17:375-382.

35. Dowsett M, Hanby AM, Laing R, Walker R, National HER2 Consultation Steering Group: HER2 testing in the UK: consensus from a national consultation. J Clin Pathol 2007, 60:685-689.

\section{Pre-publication history}

The pre-publication history for this paper can be accessed here: http://www.biomedcentral.com/1471-2407/10/534/prepub

\section{doi:10.1186/1471-2407-10-534}

Cite this article as: Tsuda et al:: HER2 testing on core needle biopsy specimens from primary breast cancers: interobserver reproducibility and concordance with surgically resected specimens. BMC Cancer 2010 10:534.

\section{Submit your next manuscript to BioMed Central and take full advantage of:}

- Convenient online submission

- Thorough peer review

- No space constraints or color figure charges

- Immediate publication on acceptance

- Inclusion in PubMed, CAS, Scopus and Google Scholar

- Research which is freely available for redistribution 